\title{
Presentación del Tema central: \\ Cuerpo, subjetividad $y$ territorialidades en resistencia
}

\section{Presentation of the Central Theme: Body, subjectivity and territoriality of the resistance}

\author{
María Cristina Fuentes Zurita ${ }^{1}$ \\ Universidad Autónoma Metropolitana Iztapalapa. \\ Ciudad de México. \\ ORCID: http://orcid.org/oooo-000I-7230-6103 \\ Claudia Piedrabita Echandía ${ }^{2}$ \\ Universidad Distrital Francisco José de Caldas. \\ Bogotá, Colombia. \\ oRCID: http://orcid.org/0000-0003-3525-1910
}

DoI: http://dx.doi.org/10.28928/revistaiztapalapa/822017/ptc/fuenteszuritamc/piedrahitaechandiacl

Los artículos que integran este Tema central: Cuerpo, subjetividad y territorialidades en resistencia, se sitúan en el contexto de la globalización actual. Esta atraviesa sus territorios y es de donde emergen los problemas de investigación y las concomitantes líneas de reflexión. Estos textos pretenden constituir un manifiesto político académico posdisciplinario, con una perspectiva ética, que vincula los procesos de subjetivación o "configuración de otras existencias", y un planteamiento epistemológico opuesto al binarismo científico.

Para afianzar lo anterior podemos decir que nos encontramos, $y$ así lo reconocemos, en el fin de la quinta etapa de la globalización. En todas, desde la primera,

\footnotetext{
1 cristina_fuentes_z@yahoo.com.mx

2،claluz7@gmail.com
} 
la española y portuguesa, iniciada en 1492 a partir del descubrimiento de América, ${ }^{3}$ han acontecido poderosas colonizaciones, con pensamientos aliados como guía, paradigmas epistemológicos con los cuales construir verdades: ideas, aparatos y técnicas científicas, muchas de ellas fabulosas y siempre con posturas ideológicas.

Después de la primera colonización siguieron: la holandesa, la inglesa, la francesa, generando la expansión del capital en el mundo. Aquellos conquistadores, fascinados con las tierras lejanas, se lanzaron por estos confines hasta hacer la América Latina, que nunca ha dejado de estar en proceso de cambio.

La crearon de la mano de grandes científicos, como ejemplo tenemos a Darwin (inglés) y Humboldt (alemán), quienes observaban las riquezas y rarezas que tenían ante sus ojos. El primero desarrollaba su teoría de la evolución al describir también a los "hombres rojos", por el color de su piel, además de la fauna y la flora, los climas. El segundo, el naturalista alemán, se interesaba en su orografía, hidrografía, recursos naturales, como el petróleo que salía a borbotones en ciertos lugares, ${ }^{4}$ siempre ayudados por los propios estudios de las élites coloniales y sus informantes locales favorecidos.

Sus aportes fueron diferentes, asombrosos y también funestos. La explotación de estas tierras y la sangre derramada se mezcló en el mestizaje, la hibridación de las culturas (en unos lugares más que en otros), siempre de maneras cada vez más sofisticadas. La concepción filosófica de Descartes, y sobre todo su pensamiento matemático, fue fundamental para el cálculo, por ejemplo, de cómo gastar menos materiales en las obras iniciadas, en la mano de obra, entre otros.

La población autóctona se diezmaba, se inició el negocio de la venta de esclavos africanos y para integrar a todos se les catequizaba, otros más terribles acababan con ellos y con los rebeldes ofreciendo recompensas por sus orejas, que en ocasiones eran arrojadas a los arroyos. Si se aceptan las metáforas, podemos decir que ocurre algo semejante en esta etapa de la globalización actual con las minas al aire libre. En los ríos cercanos se deshacen de los residuos que quedan de los cerros, de procesos químicos donde los mezclan a cielo abierto, en grandes tinajas de cemento, con ácidos, para extraer el oro. Se contaminan las aguas, se afectan los climas y por supuesto la flora y fauna de sus tierras donde se reproduce la vida. Según los datos de Consejo Nacional de Población, en el año 2010 vivían en Estados Unidos 30

3 Enrique Dussel tiene un amplio trabajo desarrollado sobre el tema (Dussel, 2014).

4 Véase literatura de sabio José Narciso Rovirosa sobre Tabasco. Biblioteca de la Universidad Iberoamericana, Ibero, plantel Ciudad de México. 
millones de mexicanos, ${ }^{5}$ que se desplazan dejando a su paso familias, problemas y se van creando esperanzas, nuevas formas de interacción y sendas desoladas. Actualmente esa cifra es posible que sea más alta.

Otros más afortunados siempre han colaborado desde la ingeniería social en las instituciones para generar mundos más racionales, tal vez más humanos, ¿̇o solo eficientes? Pero en el mundo de hoy impera la irracionalidad, los diálogos son difíciles y el encantamiento lo encontramos disfrazado de consumos falaces.

De esta quinta globalización, o etapa, iniciada a finales de los años setenta del siglo pasado, cuyo país hegemónico ha sido Estados Unidos, hemos sido actores y testigos. El capital financiero y la gigantesca revolución de las tecnologías de la información y la comunicación han sido herramientas fundamentales para su instalación y desarrollo; en tiempo y espacio. Todos conocemos los resultados que como manchas de agua se expresan en nuestros territorios, unas junto a otras simbolizan las miserias y riquezas extremas, como siempre, pero ahora de manera exponencial. Ahí, en la misma ciudad o poblado, se generan lazos de amor, de participación política, de negocios limpios o sucios, redes invisibles de comunicación, de colaboración y trabajo, incluidas las redes más eficientes que han sido las del narcotráfico y las de la muerte.

¿Y qué han hecho las ciencias, además de delinear sus propios territorios y límites? Mucho. Veamos algunos antecedentes. Mientras en América Latina existían la Inquisición y las misiones, unas con mejores resultados que otras, dependiendo de la postura que se tuvieran, en Francia, en 1777, Rousseau construía un discurso sobre una "religión cívica", con el antecedente del enciclopedismo, para lo cual se requerían nuevos sujetos: los ciudadanos.

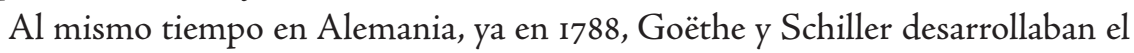
romanticismo con ayuda de la imprenta y la lectura, y el segundo, horrorizado por la sangre derramada por la revolución francesa, cuyo pensamiento había apoyado, iniciaba ese tratado ${ }^{6}$ que pretendía que la estética fuera parte de la formación ciudadana para imaginar otra forma o modo de ser. Schiller era un tránsfuga que se enfrentó al mundo aristocrático, rompió con este e invitó a escuchar al cuerpo y al corazón, sus razones, y a sentir y salir de las normas morales construidas por la realeza hipócrita, y proponía lo "auténtico". El sentir, ${ }^{7}$ liberado de esas normas para la construcción de vida, sería una vía. Tal pensamiento fue leído por los jóvenes

5 Índices de intensidad migratoria: México-Estados Unidos 2010. Consejo Nacional de Población, 2012

6 Entre varios libros está el de Cartas sobre la educación estética del hombre.

7 Pensamiento alemán precursor de los estudios de la percepción. 
judíos alemanes, futuros marxistas o heterodoxos, constructores de pensamientos diversos, ${ }^{8}$ siendo de alguna manera descolocados por pertenecer a familias de migrantes, exiliados, quienes, según Michael Löwy (2015), desde la influencia del romanticismo alemán leyeron su religión y la cábala, y construían debates, análisis y pensamientos utópicos, como la ley del valor de Marx, y el desarrollo del valor de uso y de cambio en la misma mercancía. El valor solo se extrae de la vida, y habría que reproducirla, no acabarla. La subjetividad era un recurso a resguardar, reconocer, enriquecer, ya que con ella se genera el sentido.

Así se daban rupturas y fusiones de pensamiento que invitaban a pensar diferente. Sí. Este Tema Central podría ser caracterizado como posromántico, en el sentido de provocar el pensamiento por cuenta propia, con todos los autores necesarios y el cuerpo de vuelta, ese en el que se inscriben y se guardan las inconformidades, las demandas y las potencias, es decir, las posibilidades de cambiar y así resistir. Pero es un Tema Central trans y posdisciplinario.

En esta entrega los autores invitados son de Colombia, Argentina y México. Todos son representantes de la Red Iberoamericana de Estudios Sociales (RIES) y del grupo Clacso de "Subjetividades y ciudadanías críticas, y transformaciones sociales", que reúne a nueve países y 20 universidades. Interesados en un pensamiento descolonial, ${ }^{9}$ que dé cuenta de la producción de nuevas subjetividades y territorializaciones, como parte de formas de resistencia y búsqueda de la paz. ${ }^{10}$ Se trata de un tipo de pensamiento latinoamericano que, frente a la crisis actual, se plantea críticas y ofrece, con cuidado, nuevas y renovadas metodologías desde una mirada problematizada, incluyente, amplia, actual, sin censura, ante el complejo clima político que se vive a nivel mundial. Los autores presentan investigaciones y reflexiones que analizan lo que está ocurriendo y traspasan los límites de las disciplinas, con el afán no solo de comprender sino también de comprometerse con la teoría de la acción, al igual que la teoría feminista.

Interesados en otros modos de ser y de estar, consideran que es necesario involucrarse en la construcción de un nuevo paradigma epistemológico en las

8 Desde Rosa Luxemburgo, Walter Benjamin, Franz Rosenzweig, Hannah Arendt, Ernst Bloch, Georg Lukács, Martin Buber y muchos más, pensamientos que influyeron a Freud, Marcuse, Habermas.

9 Cabe aclarar que el término descolonial hace referencia a un pensamiento que problematiza las teorías eurocéntricas que han marcado pauta en determinado país y también puede escribirse como "decolonial" al considerar o deconstruir los efectos de la colonia.

10 Este grupo se reúne frecuentemente. La primera reunión formal generó el Primer Encuentro de la RIES sobre "Pensamiento crítico y territorializaciones para la paz y la no violencia, organizado por la Universidad Autónoma Metropolitana-Iztapalapa en la Ciudad de México, en mayo de 2016. 
ciencias sociales junto con las humanidades, las ciencias biológicas, las ciencias exactas, incluyendo los campos de la estética, la ética, la política y, por supuesto, la economía.

Lejos de la competencia, es con potencia y con toda humildad que estos investigadores se han puesto frente a sí una gran tarea: la producción de una cultura de la paz y la no violencia.

El primer artículo que se presenta es el de María Cristina Fuentes Zurita, con el título "Del desencanto al encantamiento del mundo: del cuerpo con sueños al cuerpo presente", sobre un trabajo de producción de performances en el centro de la Ciudad de México con mujeres policía, trabajadoras del barrido, amas de casa, conducido por la artista Lorena Wolffer, el cual nos muestra el potencial de este método en el trabajo comunitario y de género, así como el tema de la implicación del investigador. Da cuenta de la creación de nuevas formas de producción de conocimiento social, que permiten novedosas aproximaciones al mundo en que vivimos, lo que es hoy un imperativo, al enriquecer no solo el campo de lo metodológico, sino también volver a pensar la dimensión conceptual, al acercarse a la producción estética. Desarrolla andamiajes teóricos vinculados a la cuestión, un proceso empírico de práctica performativa feminista, creando cruces con los andamiajes en el análisis de una experiencia colectiva concreta.

En segundo lugar tenemos el de Claudia Piedrahita Echandía, intitulado "Subjetivaciones poshumanas: una perspectiva ética y política". Se alimenta de las propuestas posestructurales de Gilles Deleuze y Rossi Braidotti para abordar una reflexión crítica y compleja cercana al posantropocentrismo y el poshumanismo. Da cuenta del juego posible, en el análisis de conceptos contrapuestos a la captura capitalista. Así, aborda territorializaciones/desterritorializaciones, diferencia/diferente, todo para potenciar la singularidad que, en su devenir, se fugue de esa hábil conquista de la propia diversidad por las lógicas mercantiles. Todo es posible gracias a la propuesta sobre la voluntad del poder y el materialismo vitalista, de donde deriva una metodología para estudiar y hacer actuar las cartografías existenciales. Cuerpo potenciado y subjetivaciones críticas constituyen un manifiesto ético en su trabajo.

Sigue, en tercer lugar, el texto de Oscar Useche Aldana sobre los movimientos ciudadanos de paz y resistencia, cuyo título es "Potencia, cuerpo y resistencia". Este artículo muestra claramente la preocupación por la espiritualidad cívica del ser, que se materializa en el cuerpo, continuum biológico cultural complejo (Muñiz, 20Io), que vive en sociedad. En este artículo, el autor analiza un campo relacional constituido por las fuerzas que componen los cuerpos, los dispositivos que el poder ha generado para capturar e incorporar su potencia y las resistencias que emergen 
como líneas de fuga de la dominación. Para Useche, el cuerpo no es independiente de sus relaciones y sus cambios y movimientos que se dan en la tensión entre servidumbre voluntaria y des-sujeción, que solo puede ser producida por la resistencia.

Finalmente, se presenta la investigación de Pablo Vommaro sobre los jóvenes latinoamericanos en resistencia,"Territorios y resistencias: configuraciones generacionales y procesos de politización en Argentina". Este autor aborda ciertos movimientos de jóvenes entre cuyas formas de expresión involucran los afectos, con sus cuerpos como vehículos que se movilizan en lo local. Es un aporte relevante sobre la política como producción territorial y el territorio como producción política, al articular el papel de las generaciones y del barrio. ${ }^{11}$ Muestra este proceso contextualizado en el caso de dos colectivos juveniles argentinos: uno de alcance provincial y otro nacional.

Estos artículos son eminentemente críticos respecto de los efectos de la globalización contemporánea; no solo los describen y analizan, también pretenden salir al encuentro con un pensamiento posdisciplinario. Para ello, tejen un andamiaje conceptual de pensamientos potentes, desde diversos confines del mundo: de occidente, oriente, y también una postura ética al considerar el precepto africano: "Yo soy porque tú eres y tú eres porque yo soy". Se reconocen parte de los dispositivos de la subjetividad y el poder simbólico y hacen un llamado a revisar los modos o formas bajo los que se produce el conocimiento social y académico, para no solo repetir o contribuir a la competitividad.

Estos textos muestran la aspiración de renunciar a epistemologías limitadas que estrechan la mirada, $y$ a las subjetividades que producen cuerpos para la guerra, para la enfermedad, para la opresión; que la resistan y creen condiciones de vida consciente de ellos y de los seres no humanos. Para ello, parten de apuestas teóricas y metodológicas situadas, rompen con epistemologías del binarismo que no consideran que las emociones y el poder interactúan en los cuerpos. El modo binario antepone la naturaleza y la ciencia a la cultura, lo objetivo a lo subjetivo, el pensamiento a la acción, la mente al cuerpo, lo masculino a lo femenino, introduciendo jerarquizaciones al pensamiento social, el cual no solo hay que estudiar sino también transformar, más en estos tiempos.

11 En México ese espacio es la escuela, según los expertos. 


\section{Referencias bibliográficas}

Dussel, Enrique

201416 Tesis de economía politica. Interpretación filosófica, México D. F., Siglo xxi Editores.

Lowy, Michael

2015 Los judios heterodoxos. Romanticismo, mesianismo, utopía. México: Antrophos-Universidad Autónoma Metropolitana-Iztapalapa.

Muñiz, Elsa

2010 Disciplinas y practicas corporales: una mirada a las sociedades contemporáneas, Barcelona: Anthropos-Universidad Autónoma Metropolitana-Azcapotzalco. 